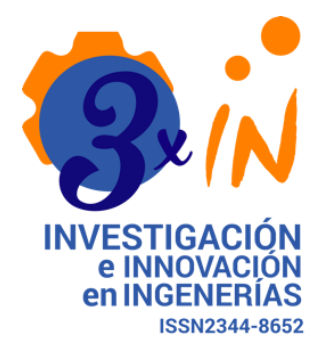

Open Access

Recibido:

31 de octubre de 2019

Aceptado:

12 de febrero de 2020

Publicado:

13 mayo de 2020

Correspondencia:

josemunive@infotephvg.edu.co

DOI:

https://doi.org/10.17081/invinno.8.1 .3409

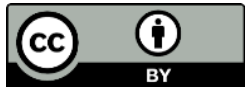

(c) Copyright: Investigación e Innovación en Ingenierías

\section{Calidad de la iluminación en las aulas de clase en una Institución de Educación Superior}

\section{Quality of lighting in classrooms at an Institution of Higher Education}

\author{
José Martin Munive Álvarez \\ Instituto Nacional de Formación Técnica Profesional Humberto Velázquez García, \\ Colombia
}

\section{Resumen}

Objetivo: Determinar la calidad de la iluminación y las estrategias de mejoramiento en las aulas de clase en una institución de educación superior. Metodología: El estudio fue descriptivo, se realizó la medición de la iluminancia en las aulas, así como también la uniformidad de la iluminancia, además se evaluó Índice de deslumbramiento, Índice general de rendimiento del color y temperatura colorimétrica nominal por cada lámpara. Las mediciones ambientales o luxometria se realizaron con un luxómetro marca extech en un total de 15 aulas en las tres jornadas en que se labora. Resultados: la iluminancia en la mañana y tarde cumplen la mayoría de las aulas, contrario en la jornada de la noche en donde ninguna cumple con la norma ISO 8995, además la mañana y la tarde existen aulas con niveles excesivos de iluminación y la noche con niveles muy por debajo de los apropiados, la mayoría de las aulas en las tres jornadas no cumplen con la uniformida, no existe deslumbramiento molesto, así como también algunas lámparas no cumplen con rendimiento del color apropiado, y la temperatura colorimétrica requerida. Conclusiones: Existen aulas que no cumplen con la iluminancia, así como con la uniformidad en las tres jornadas, se resalta que hay un aula que no cumple con tres parámetros, iluminancia, uniformidad y rendimiento del color, esto puede afectar el desempeño laboral y ocasionar molestias y enfermedades en la vista.

Palabras claves: Iluminancia, uniformidad, niveles de iluminación, Índice de deslumbramiento, calidad de la iluminación.

\begin{abstract}
Objective: To determine the quality of lighting and strategies for its improvement in classrooms at a higher education institution. Methodology: The study was descriptive. Measurements of the lighting in the classrooms and the uniformity of the lighting were conducted. In addition, glare index, general color performance index, and nominal color temperature for each lamp were assessed. Environmental or illuminance measurements were conducted with an Extech luxmeter in a total of 15 classrooms for three workdays at the institution. Results: The illumination in the morning and afternoon meets the standards in most classrooms. In contrast, in the evening, none of them meets the ISO 8995 standard. Besides, in the morning and afternoon, there are classrooms with excessive levels of lighting and in the evening, with levels far below the appropriate ones. Most classrooms analyzed in the three workdays did not meet the standard; there is no annoying glare, and also some lamps do not meet the appropriate color performance and the required colorimetric temperature. Conclusions: There are classrooms that did not meet illumination standards and uniformity in the three workdays. It is highlighted that there is a classroom that does not comply with three parameters: illumination, uniformity, and color performance. This can affect work performance and cause discomfort and eye disorders.
\end{abstract}

Keywords: Illumination, uniformity, lighting levels, glare index, lighting quality.

Como citar (IEEE): J.M. Munive - Álvarez, "Calidad de la iluminación en las aulas de clase en una Institución de Educación Superior", Investigación e Innovación en Ingenierías, vol. 8, nº. 1, 2020. DOI: https://doi.org/10.17081/invinno.8.1.3409 


\section{Introducción}

El ser humano tiene una habilidad sorprendente para adaptarse a su medio ambiente y a su entorno inmediato. Hay muchos tipos de energía que pueden utilizar los humanos, la luz es la más importante. La radiación lumínica es un elemento esencial de nuestra capacidad interactuar con el entorno y ver todo lo que nos rodear, además necesaria para identificar la forma, el color y la perspectiva de los objetos que nos rodean a través del sentido de la visión. La mayoría de la información que obtenemos a través de los sentidos la obtenemos a través de los ojos (cerca del 80\%). Y al estar tan familiarizados a disponer de ella, consideramos por supuesta su labor. Sin embargo, no debemos olvidar que ciertos aspectos del confort humano, como nuestra salud mental y física o nuestro nivel de fatiga, se ven perjudicados por la falta o excesiva iluminación en los entornos en que desempeñamos nuestras funciones y por la percepción del color de las cosas que nos rodean debido a características de la anatomía del ojo como son la acomodación y la adaptación.

Desde el punto de vista de la seguridad y salud en el trabajo, el rendimiento y el confort visual es excepcionalmente importante, debido a que un gran porcentaje de accidentes de trabajo se deben, entre muchas razones, a insuficiencia en la iluminación o a fallas cometidas por el trabajador, a quien le resulta complicado reconocer materiales u objetos o los riesgos asociados con el mobiliario maquinaria, herramientas, medios de transporte $y$ recipientes peligrosos. $[1,2,3,4,5]$

El ser humano percibe toda la información mayoritariamente por los sentidos siendo uno de los principales para ajustarse a su entorno es la visión, ya que permite la posibilidad de diferenciar los objetos, cuya apreciación está directamente enlazada con la proporción de luz que ellos reflejan o transmiten. Si la iluminación es deficiente, se distinguen mal los objetos, se detectan bajos rendimientos laborales, y se pueden ocasionar accidentes graves en personas y/o daños en equipos o materiales. Por el contrario, si la iluminación es la correcta, se revelan los contornos, se hace más sencillo visualizarlos, se disminuye el cansancio visual, se eleva la moral, se facilita la inspección y se reducen los accidentes además está demostrado que un mejora en la iluminación de 300 a 500 lux eleva la productividad en un $8 \%$ y una mejora de 300 lux a 2000 lux incrementa la productividad en un $20 \%$. $[6,7,8]$

La iluminación correcta y reflexión adecuada del ambiente, además de otros parámetros de la calidad de la iluminación como son la uniformidad, el índice de deslumbramiento y el rendimiento del color apropiados permiten al hombre, en condiciones confortables de confort, desempeñar su trabajo de forma más segura y alto rendimiento en el trabajo, ya que incrementa la visibilidad en el entorno del trabajo y permitiendo vigilar mejor el medio 
ambiente laboral utilizado. Por ello debe ser ajustada antes de poner en marcha el proyecto y conservarla posteriormente por los servicios de mantenimiento de la organización. $[9,10]$.

La deficiencia en las condiciones de iluminación presente en los establecimientos de trabajo están generando una de las molestias más comunes entre los trabajadores colombianos que es la fatiga visual, además potencializa otros riesgos psicolaborales, de seguridad y crea un disconfort ergonómico.

Las malas condiciones de iluminación es la principal causa que influyen de modo revelador en las afecciones de la vista. Los rangos de iluminación indicados están entre los 300-500 lux, además para las oficinas fluctúan desde 150 hasta 300 lux. Una equivocada colocación de luminarias en el campo visual y el plano de trabajo provocaría encandilamiento o deslumbramientos que produciría fatiga ocular. Estos deslumbramientos pueden ser ocasionado por un emplazamiento imperfecto del mobiliario de la oficina. [11].

En las instituciones de educación superior se estableció que el $74 \%$ de los salones de clase de postgrados cumple con los requisitos mínimos de iluminación, el $26 \%$ no cumple con la normatividad legales, además existen aproximadamente $25 \%$ de puntos críticos, en donde se deben establecer o implementar controles para atenuar el riesgo por iluminación, las aulas de postgrado se construyen para evitar el ingreso de la luz solar, ya que estas cuentan con sistemas de proyección de datos es decir el uso de video vean, es importante indicar que los estudiantes y docentes no están expuestos a más de ocho horas a este ambiente de trabajo. [12].

En aulas de una universidad se demostró, que estas no cumplen con el requisito de la norma, ya que las luminarias que están no cumplen con los lúmenes deseados, tampoco cumplen con la cantidad de luminarias que deben tener cada una de ellas [13].

La iluminación de un aula de clases debe ser adecuada para todas las actividades que intervienen en el proceso de enseñanza aprendizaje, como lo son la escritura, lectura de libros y uso del tablero. Como estas actividades son similares a las de las oficinas, los circunstancias generales de iluminación pueden aplicarse a las escuelas.

En una institución prestadora de salud en Bogotá se hizo una investigación que muestra las afectaciones por las malas condiciones de iluminancia, después de realizar la evaluación optométrica laboral se llegó a la conclusión que el $56 \%$ de los trabajadores usa algún tipo de corrección de la visión. El $89 \%$ de la población trabajadora de la IPS presentan algún tipo de indicio que según la literatura puede estar conectado con un disconfort por 
iluminación en el desarrollo de las distintas labores. Aunque los trabajadores pueden presentar más de un afección, se identifica que el $22 \%$ de los trabajadores han tenido cansancio ocular en el transcurso de sus labores, el $34 \%$ enrojecimiento eventual, el $22 \%$ incomodidad a la luz solar, el $11 \%$ molestia a la luz artificial y el $34 \%$ sombras en la visión .

Al llevar a cabo el estudio externo al ojo derecho se mostró que el $56 \%$ presentan Hiperemia, Pingüecula o Pterigión, al mirar al ojo izquierdo el $44 \%$ presenta alguna de las condiciones anteriores. Al determinar la visión de color, tanto en ojo derecho como en ojo izquierdo de identifica que el $11 \%$ presenta una anormalidad en este sentido.

El $89 \%$ de la población muestra alguna deficiencia refractiva, en mayor cantidad el astigmatismo, le sigue la presbicia y para finalizar la miopía, además se tiene certeza que el $50 \%$ de los lugares de trabajo no cumplen con los niveles mínimos de iluminación establecidos en el Reglamento Técnico de iluminación y alumbrado público [14].

Además, se puede inferir que en las empresas no se están implementando medidas de intervención, esto se evidencia en un estudio hecho en una petrolera en Venezuela, que demuestra que la distribución de las luminarias es irregular, gran parte de los lugares evaluados en la empresa no cumplen con los niveles de iluminancia establecidos en la norma vigente, y además es un factor preponderante para la calidad de la iluminación. Es un hecho que los niveles de iluminación no son los adecuados en las empresas y afectan a las personas esto se puede comprobar por estudios de Calidad de iluminación en ambientes de trabajo.

Se analizaron un total de 1.015 puestos de trabajo, de los cuales 761 de ellos presentaban niveles de iluminación por debajo de 500 lux. Esto nos conlleva a que el $74,98 \%$ de los trabajadores realizan sus actividades en estos puestos, se encontraban en situación de riesgo alto por iluminación. La empresa cuenta con un número total de luminarias y lámparas instaladas en las oficinas analizadas de 1.495, de las cuales 260 están defectuosas y 14 en mal funcionamiento, lo que representó el $17,39 \%$ y $5,38 \%$ respectivamente. $[15,16,17]$

Es una condición demostrada que los requisitos de iluminación no son los apropiados en las organizaciones y afectan a los empleados, se puede deducir por varios estudios que entre el $30 \%$ y $40 \%$ de las áreas de las organizaciones tienen muy baja iluminación llegando a estar por debajo de los 200 lux, usualmente se observa que existe una deficiencia de aprovechamiento de la luz solar, siendo los sistemas artificial sean fluorescente $o$ incandescente, la esencial fuente de iluminación en los espacios de trabajo. El aprovechamiento de la iluminación natural en los recintos de trabajo no se dirige únicamente al ahorro o a la sustitución de 
recursos naturales, sino a la mejora de las condiciones de calidad de vida con el menor gasto de todos los recursos, ya sean de índole financiero y energéticos. El propósito es conseguir el más importante bienestar en el trabajo con el menor costo disminuirá el consumo de combustibles convencionales y también mejorará la calidad de vida de los habitantes. $[18,19,20]$.

\section{Metodología}

Se realizó un estudio descriptivo que describen y evalúan las variables que influyen en la calidad de la iluminación, fue realizado en ciénaga magdalena en el instituto técnico profesional Humberto Velázquez García en el año 2017, el trabajo de campo se realizó en los meses de junio y julio, la población está conformada por 15 aulas de clase que se utilizan en tres jornadas, mañana, tarde y noche, que se encuentran distribuidas en dos edificios y en tres pisos. . Para el cumplimiento de los objetivos primero se hizo un diario de campo en donde se anotó lo observado, después se procedió a realizar la medición de iluminancia media, uniformidad de la iluminancia, se recolecto la información para definir los índices de deslumbramiento y rendimiento del color, estos parámetros son comparados con la ISO 8995 y el reglamento técnico de iluminación y alumbrado público RETILAP resolución número no 181331 del ministerio de minas y energía. Se utilizó el método de la cuadricula, primero hallamos la dimensión de cada salón por aula, largo, ancho, altura de la luminaria al plano de trabajo, constante del salón y los puntos mínimos de muestreo por aula y se procedió hacer la medición con un Luxómetro digital marca EXTECH modelo 407020 en las tres jornadas en días soleados.

\section{Resultados}

A continuación, se presentan los resultados obtenido con los diferentes instrumentos.

\section{Iluminancia media}

En la jornada de la mañana el $80 \%$ de las aulas cumplen con los niveles de iluminancia requeridos, mientras que el $20 \%$ no cumplen con los niveles de iluminancia requeridos, las aulas 209, 210 y 212 no cumplen, además se observan niveles excesivos de iluminancia en las aulas 204, 207, 208, 301 que son perjudiciales para la salud (Figura 1). 
Figura 1. Iluminancia total mañana

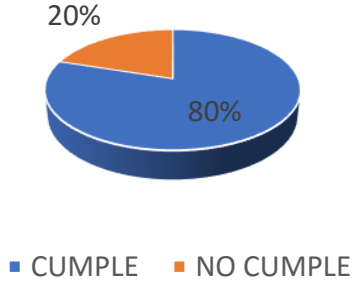

Fuente: Elaboración propia

En la jornada de la tarde cumple el $86.6 \%$ de las aulas con los niveles de iluminancia requeridos, mientras que $13,4 \%$ no cumplen con los niveles de iluminancia requeridos representado en las aulas 210 y 212, además se observan niveles de iluminancia excesivos en un 33.3\% en las aulas 203, 204, 206, 214, 301 que son perjudiciales para la salud (Figura 2).

Figura 2. lluminancia jornada tarde

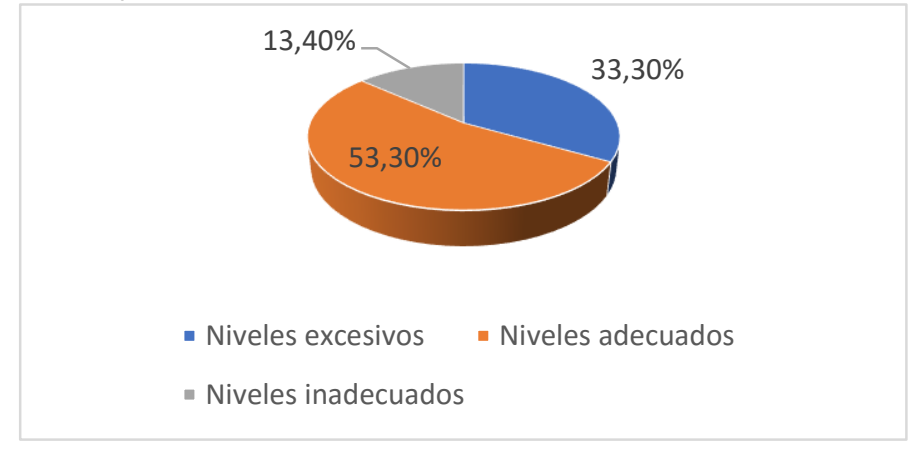

Fuente: Elaboración propia

En la jornada de la noche no cumple el $100 \%$ de las aulas con los niveles de iluminancia requeridos, llama la atención que el 93.3\% de las aulas tienen iluminancias por debajo de 200 lux que equivale a un aproximado de $40 \%$ por debajo de la iluminancia requerida.

\section{Uniformidad de la iluminancia}

En la jornada de la mañana el $6,6 \%$ de las aulas cumplen con la uniformidad requerida, mientras que el $93,3 \%$ no cumplen con la uniformidad, esto quiere decir que una sola aula en la jornada de la mañana cumple con la uniformidad, siendo el aula 204 (Figura 3). 
Figura 3. Uniformidad en la jornada mañana

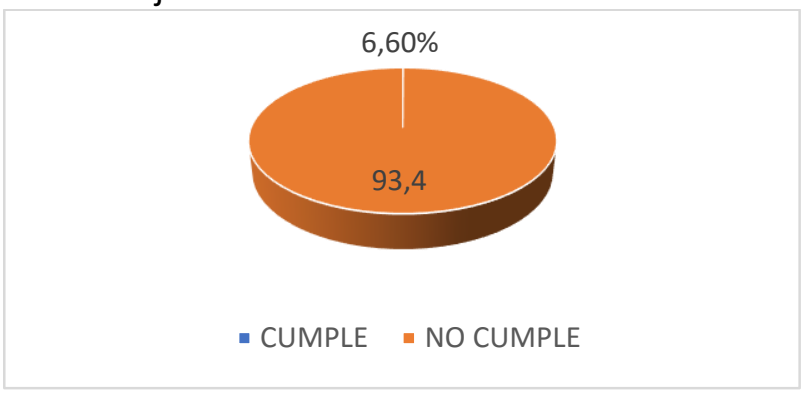

Fuente: Elaboración propia

En la jornada de la tarde el $100 \%$ de las aulas no cumplen con uniformidad requerida, además en la jornada de la noche el $26,6 \%$ de las aulas cumplen la uniformidad requerida, mientras que el $73,3 \%$ no cumplen con los niveles de uniformidad, esto quiere decir que 4 aulas en la jornada de la noche tienen uniformidad requerida, las aulas, 202, 203, 205, 206.

\section{Rendimiento Del Color}

El $73.4 \%$ de las lámparas colocadas en las aulas cumple con el índice de rendimiento del color y el $26.6 \%$ no cumplen con este parámetro y son las aulas 210, 213, 214, 301.

\section{Índice de deslumbramiento}

En las aulas de la institución no existe deslumbramiento molesto por dos aspectos importantes, el primero que las luminarias no se encuentran en un ángulo de 45 grados con respecto a la línea de visión del observador, el segundo es que las luminarias están montadas a una gran altura y al hacer los cálculos las dimensiones del salón no se ajustan a las tablas propuestas por CIE 117: 1995 Disconfort glare in lighting y en el informe CIE 1902010 Cálculo y presentación de las tablas del Índice Unificado de Deslumbramiento para las luminarias en iluminación de interiores.

\section{Discusión}

De acuerdo con el instituto nacional de seguridad higiene del trabajo de España, entre las diferentes actividades que realizan las personas en el transcurso de su vida, una de las que ocupa un porcentaje de tiempo importante, no sólo en el periodo de tiempo sino también en el área o espacio, es el trabajo. En este orden de ideas la actividad ocupacional, para que pueda transcurrir de una manera eficaz y eficiente, es imprescindible que la luz (propiedad ambiental) y la visión (cualidad personal) se complementen, debido a que se considera que el $50 \%$ de la información sensorial que percibe el hombre es de tipo visual, es decir, tiene como origen básico la luz. 
Al analizar varios tipos de investigaciones con relación a la calidad de la iluminación nos podemos dar cuenta que en la totalidad de ellas, el grado de iluminación en los espacios de trabajo no son los convenientes infligiendo los estándares nacionales de cada país e internacionales, esto se ve evidenciado en este estudio en donde en las tres jornadas existen aulas que no tienen la iluminancia requería sobre todo en la jornada de la noche, además la uniformidad no es la adecuada, esto se puedo a demostrar en una investigación titulada Lighting for work: visual and biological effects, que confirman que los fundamentales criterios de calidad son la iluminancia, la uniformidad, el deslumbramiento y el rendimiento del color.

En la institución se presentan anomalías en la iluminación, argumento por el cual los empleados deben hacer mayor esfuerzo para no ver afectado su normal desempeño, está demostrado que un mejora en la iluminación de 300 a 500 lux acrecienta el rendimiento en un $8 \%$ y una mejora de 300 lux a 2000 lux incrementa el rendimiento laboral en un $20 \%$. Es necesario que se tomen acciones inmediatas cada vez que se observe alguna condición por debajo del estándar propuesto por la literatura o por la normatividad colombiana, debido a que una demora en la toma de acciones afecta la salud y la seguridad del trabajador.

\section{Conclusiones}

En la jornada de la mañana y la jornada de la tarde las aulas cumplen en su mayoría en más del $80 \%$ respectivamente con los niveles de iluminancia requeridos por la norma ISO 8995 2002, debido a que la iluminación es mixta, compuesta por iluminación artificial con lámparas fluorescentes y natural debida a que cuenta con una ventanas de gran dimensión.

Otro aspecto relevante es que 4 aulas en la jornada de la mañana presentan niveles excesivos de iluminancia, así como 5 en la jornada de la tarde que presentan el mismo problema, debido a la iluminación natural producidas por el sol, siendo un aula la que presenta este inconveniente en las dos jornadas.

En la jornada de la noche todas las aulas no cumplen con la iluminancia requerida, además los niveles de iluminación están por debajo de 200 lux, que representa aproximadamente el $40 \%$ muy por debajo de la iluminancia requerida.

Con relación a la uniformidad en la jornada de la mañana y la tarde no cumplen con $93.3 \%$ y $100 \%$ respectivamente, además en la jornada de la noche existe menos incumplimiento con un $73.3 \%$, podemos concluir que la mayoría de aulas aulas en las tres jornadas no cumplen con la uniformidad de la iluminación, la mayoría de las lámparas cumplen con el índice de rendimiento del color y no existe deslumbramiento molesto. 


\section{Referencias bibliográficas}

1. Instituto Nacional de Seguridad e Higiene en el Trabajo, Capítulo 46 Iluminación. Madrid: enciclopedia de la OIT, 2012.

2. Superintendencia de riesgos en el trabajo. [www.srt.gob.ar]. Ministerio de trabajo, empleo y seguridad social; c2011 [actualizada 20 feb. 2017; consultado 06 marzo 2017]. Disponible en: http://www.srt.gob.ar/adjuntos/prevencion/guiailuminacion.

3. F. Henao - Robledo. Riesgos físicos II: iluminación. Bogotá: Ecoe Ediciones, 2007.

4. D. Benavides, Caracterización y evaluación de iluminancia en dos áreas de trabajo. Incidencia de la calidad de iluminación en términos de riesgo que afectan la seguridad del trabajador en dos empresas del sector eléctrico Tesis [Internet]. 2014 [citado el 5 de Marzo de 2017]. Recuperado a partir de: http://www.bdigital.unal.edu.co/48484/1/52958054.2015.pdf

5. Manual de higiene industrial. Fundación MAPFRE $3 \underline{a}$ ed Publicación. Madrid: Editorial MAPFRE, 1996

6. G. López, Análisis del sistema de iluminación. Córdoba: El Cid, 2009

7. M. de Carli, D. Giuli, R. Zecchin, "Review on visual comfort in office buildings and influence of daylight in productivity", Indoor Air, pp. 17-22. 2008.

8. S. Sanaz, "The Impact of Indoor Lighting on Students' Learning Performance in Learning Environments: A knowledge internalization perspective", International Journal of Business and Social Science, vol. 3, $\mathrm{n}^{\circ}$. 24, pp. 127-136., 2012.

9. J.M. Cortez, Técnicas de prevención de riesgos laborales: seguridad e higiene del trabajo, México: Tebar Ediciones, 2017.

10. M. Bovea Edo, Manual de seguridad e higiene industrial para la formación en ingeniería, 2011.

11. V. B. Wout and V den B. Gerrit, " Lighting for work: visual and biological effects. April 2003, Philips Lighting, The Netherlands, Revista Lighting Research and Technology", 2003.

12. G. Aldo y G. Montes, "Ergonomía ambiental: Iluminación Y Confort Térmico En Trabajadores De Oficinas Con Pantalla De Visualización De Datos", Revista de ingeniería, matemáticas y ciencias de la información, 2014. 
13. A. Argudo - Garzón y D. Ponce Altamirano, Gender in visions of the daughters of albion and La Emancipada: a comparative analysis of the problem of gender in the Ecuadorian and british romantic societies. Tesis [Internet]. 2011 [citado el 5 de marzo de 2019]. Recuperado a partir de: http://dspace.ucuenca.edu.ec/handle/123456789/2089

14. Reglamento Técnico de lluminación y Alumbrado Público. Resolución número no 18 1331. Boletín Oficial del Estado, (0608-2009).

15. J. Beltrán - Molina y C. Merchán - Arévalo, “Niveles de iluminación y su relación con los posibles efectos visuales en los empleados de una IPS de Bogotá", Revista Movimiento Científico, vol. 7, $\mathrm{n}^{\circ} .1$, 31-37., 2013.

16. M. Corredor, E. Cabeza y M. Sanchez, "Evaluación de los Riesgos por lluminación en los Puestos de Trabajo de Oficinas PDVSA a través de un Programa de Computación", Revista Multidisciplinaria del Consejo de Investigación de la Universidad de Oriente, vol. 12, $\mathrm{n}^{\circ}$. 48, 2008.

17. Ministerio de salud. Instituto de salud pública. protocolo para la evaluación de la luminancia e iluminancia en los lugares de trabajo. Santiago de chile: departamento de salud ocupacional; 2008.

18. H.V. Anacleto, "Calidad De Iluminación En Ambientes De Trabajo De La Dirección General De Salud Ambiental. Universidad Nacional Mayor de San Marcos". http://cybertesis.unmsm.edu.pe/handle/cybertesis/2502

19. A. Cremus y J. Mangosio, Seguridad e higiene en el trabajo : Un enfoque integral. Buenos Aires : Alfaomega, 2011.

20. M.D. Faustino, Higiene industrial: manual para la formación del especialista (7a ed.). España: Lex Nova., 2008. 